\title{
c-Myc modulates glucose metabolism via regulation of miR-184/PKM2 pathway in clear-cell renal cell carcinoma
}

\author{
JIWEI HUANG, WEN KONG, JIN ZHANG, YONGHUI CHEN, WEI XUE, DONGMING LIU and YIRAN HUANG \\ Department of Urology, Renji Hospital, School of Medicine, Shanghai Jiao Tong University, Shanghai 200127, P.R. China
}

Received February 28, 2016; Accepted June 23, 2016

DOI: 10.3892/ijo.2016.3622

\begin{abstract}
Renal cell carcinoma (RCC) is one of the most malignant tumors worldwide. Among all subtypes of RCC, clear-cell RCC (ccRCC) is the most common and aggressive one. The difficulty in overcoming resistance of traditional treatment is a threat for ccRCC therapies. Therefore, to understand the mechanism that underlies ccRCC progression is critical for new drug development. In the present study, we identified that miR-184 could be downregulated by c-Myc, which is different from the standard opinion that c-Myc is a target of miR-184. Overexpression of pre-miR-184 changed the metabolic and proliferation features of ccRCC cells by reducing cell glucose consumption, lactate production and cell proliferation. Further analysis by computer bioinformatics revealed that PKM2 is a target of miR-184. Both PKM2 mRNA and protein were significantly affected by addition of miR-184. Importantly, the PKM2 expression level was indeed increased in ccRCC samples, which is totally reverse compared to the decreased miR-184 expression level. Interestingly, we found that when PKM2 was knocked down in ccRCC cells, the rapid proliferation, high glucose consumption and high lactate production were all clearly inhibited, which indicates metabolic reprogramming and cancer progression blocking the in ccRCC cells. Our findings shed new light on ccRCC molecular study and provide a new and solid basis for developing ccRCC therapy.
\end{abstract}

\section{Introduction}

Renal cell carcinoma (RCC) is identified as the seventh most common cancer for adult humans and account for $2 \%$ of all cancers worldwide $(1,2)$. The lack of efficient clinical diagnostic and curative plan is one of the major reasons for RCC lethality $(3,4)$. Among all the subtypes of RCC, clear-cell renal cell carcinoma (ccRCC) is the most frequently discovered

Correspondence to: Dr Yiran Huang, Department of Urology, Renji Hospital, School of Medicine, Shanghai Jiao Tong University, 160 Pujian Road, Pudong, Shanghai 200127, P.R. China

E-mail: yrhuangrenji@163.com

Key words: c-Myc, miR-184, PKM2, clear-cell renal cell carcinoma, glucose metabolism subtype. Patients with ccRCC usually have the lowest probability of recovery compared with other RCC subtypes (5).

ccRCC is a metabolic disease characterized by the dysregulation of many metabolic pathways, including abnormal oxygen and energy consumption, as well as aberrant nutrient sensing (6). During ccRCC progression, inactivation of certain genes (such as von Hippel-Lindau, VHL) and signaling pathway activation (such as MYC pathway) were reported to take part in the abnormal metabolic profiles (7-10). Recently, a large number of abnormally expressed miRNAs were reported to be involved in the progression of ccRCC (11). Among these miRNAs, miR-184 is widely dysregulated in various human cancers (12-15). Previous studies have demonstrated that miR-184 was downregulated and functions as a tumor suppressor in RCC $(16,17)$. However, the molecular mechanism of miR-184 in regulating RCC progression is still unknown. It is worth mentioning that c-Myc, a wellknown oncogene that promotes cell cycle, cell differentiation and apoptosis (18), is reported to be a target of miR-184 in many human malignancies, including non-small cell lung cancer $(19,20)$, nasopharyngeal carcinoma (14), tongue squamous cell carcinoma (12) and hepatocellular carcinoma (15). Notably, the expression of c-Myc was indeed upregulated in ccRCC $(10,21)$. However, whether downregulated miR-184 is related to upregulated c-Myc in ccRCC is still unknown.

In this study, we indentified PKM2 as a new target of miR-184. Furthermore, the expression of miR-184 can be negatively regulated by a well-known oncogene c-Myc. PKM2 is highly expressed in human ccRCC samples. Remarkably, PKM2 knockdown leads to metabolism reprogramming and growth inhibition in ccRCC cell lines.

\section{Materials and methods}

Patient samples. Fifty pairs of human ccRCC and normal kidney samples were collected from our institution, where the samples were evaluated by a comprehensive clinical assessment. All the paraffin-embedded sections and frozen samples used in the present study were approved by ethics license of RJ2014N017.

Cell culture and transient transfection. The 786-O and RCC4 cell lines were from the American Type Culture Collection (ATCC; Manassas, VA, USA). The basic medium for 786-O and RCC4 cells were RPMI and Dulbecco's modified Eagle's 
medium (DMEM) supplemented with $2 \mathrm{mM}$ glutamine, respectively. The culture medium consisted of basic medium supplemented with $10 \%$ fetal bovine serum (FBS), 1X penicillin/streptomycin (both from Invitrogen, Grand Island, NY, USA). Cells were incubated at $37^{\circ} \mathrm{C}$ in a humidified atmosphere of $5 \% \mathrm{CO}_{2}$ and $95 \%$ air. For transfection, cells were plated subconfluently onto each well of 24-well tissue culture plates $24 \mathrm{~h}$ before transfection. Transient transfection of pre-miR-184 or control plasmid at a final concentration of $20 \mathrm{nM}$ per well was accomplished with Lipofectamine 2000 (Invitrogen) according to the manufacturer's protocol. The human PKM2 siRNA and control siRNA were purchased from OriGene. The human c-Myc plasmid was generated according to standard molecular biology techniques and verified by sequencing. c-Myc plasmids were transfected with Lipofectamine 2000 according to the manufacturer's instructions.

RNA isolation and miRNA detection. Total RNA of cells and tissues were extracted using TRIzol reagent (Invitrogen) according to the manufacturer's instructions. For isolation of small RNAs the mirVana miRNA isolation kit (Ambion) was used. Detecting the miRNA mature form was performed using the mirVana qRT-PCR miRNA detection kit (Ambion). qRT-PCR primer sets were according to the manufacturer's instructions. We chose U6 small nuclear RNA as an internal control. The real-time RT-PCR assays were performed using the 7500 Fast Real-Time PCR System for quantitative mRNA detection and with iTaq Fast SYBR-Green Supermix (Bio-Rad Laboratories). The primers for real-time PCR were human actin: 5'-AAGGAGCCCCACGAGAAAAAT-3' (forward) and 5'-ACCGAACTTGCATTGATTCCAG-3' (reverse); human c-Myc: 5'-TCAAGAGGTGCCACGTCTCC-3' (forward) and 5'-TCTTGGCAGCAGGATAGTCCTT-3' (reverse); human PKM2: 5'-ATAACGCCTACATGGAAAAGTGT-3' (forward) and 5'-TAAGCCCATCATCCACGTAGA-3' (reverse).

MTT experiments. Cells were seeded in 96-well microtitre plates with $1 \times 10^{3}$ cells/well and incubated for $24 \mathrm{~h}$ in $100 \mu \mathrm{l}$ culture medium. MTT [100 $\mu \mathrm{l}(5 \mathrm{~g} / \mathrm{l})]$ was added to the cells, which were then cultured for a further $4 \mathrm{~h}$. Following the removal of the supernatant fluid, $100 \mu \mathrm{l} /$ well DMSO was added to the cells which were agitated for $15 \mathrm{~min}$. The absorbance was measured at $570 \mathrm{~nm}$ by a microplate reader. Each assay was repeated three times.

Metabolism assays. 786-O and RCC4 cells were seeded into 24-well plate with $200 \mathrm{ml}$ of medium in each well. To determine the levels of glucose and lactate, the supernatants of cell culture media were collected and assayed for glucose and lactate levels by using glucose assay kit and lactate assay kit (BioVision Inc., Milpitas, CA, USA) according to the manufacturer's instructions. Glucose consumption and lactate production were calculated based on the standard curve, and normalized to the cell number.

Colony formation assay. Anchorage-independent growth was determined by the ability of cells to form colonies in soft agar. 786-O and RCC4 cells were diluted in fresh medium, reseeded in $0.3 \%$ agar in 6 -well plates. Cell growth was allowed to proceed for 25 days. Formed colonies were fixed with $10 \%$ formaldehyde and counted using the Col Count instrument (Oxford Optronix Ltd., Abingdon, UK).

Luciferase reporter assay. Luciferase assay was performed as previously described (22). Briefly, 786-O cells of 50\% confluence in 24-well plates were transfected with FuGENE HD reagent (Roche). In each well, 200 ng pRL-TK-PKM2-3'-UTR reporter firefly luciferase construct was co-transfected with $1 \mathrm{ng}$ Renilla plasmid and pre-miR-184, or Ctrl microRNA (Ambion) into cells. Cell extracts were prepared $48 \mathrm{~h}$ after transfection and luciferase activity was measured with the Dual-Luciferase reporter assay system (Promega). PKM2 3'-UTR-luciferase or PKM2 3'-UTR-luciferase mutant activities were normalized by first dividing the luciferase activity in each well by Renilla activity.

Western blot analysis. Lysates were prepared from $1 \times 10^{7}$ cells by dissolving cell pellets in $100 \mu \mathrm{l}$ of lysis buffer containing protease inhibitors. Lysates were centrifuged at $18,000 \mathrm{x} \mathrm{g}$ for $15 \mathrm{~min}$ and the supernatant was collected. The protein concentration was estimated with the Bio-Rad protein assay kit (Bio-Rad Laboratories, Hercules, CA, USA) using bovine serum albumin as a standard. Sample proteins were resolved by $10 \%$ sodium dodecylsulfate polyacrylamide gel (Bio-Rad Laboratories) electrophoresis and then electrophoretically transferred to PVDF membrane (Millipore) and blocked with 5\% BSA (Sigma-Aldrich). Subsequently the primary antibodies PKM2 (1:1,000; Cell Signalling Technology), c-Myc (1:2,000; Santa Cruz Biotechnology) and actin (1:10,000; Sigma-Aldrich) were added. After overnight incubation at $4^{\circ} \mathrm{C}$ the blots were washed, exposed to HRP-conjugated corresponding secondary antibodies for one hour and finally were visualized by ECL Advanced Solution (GE Healthcare Life Sciences). Digital images were captured by Gel Doc ${ }^{\mathrm{TM}}$ gel documentation system (Bio-Rad Laboratories).

Immunocytochemistry and in situ hybridization. The procedures of immunohistochemical studies for paraffin-embedded sections were performed as previously described (23). Antihuman PKM2 antibody (1:100; GeneTex Inc.) and anti-human c-Myc antibody (1:100; Santa Cruz Biotechnology) were used as primary antibody. miR-184 in situ hybridization assay was conducted by using locked nucleic acid (LNA)-digoxigenin (DIG)-labeled probes (Exiqon, Vedbæk, Denmark) as previously described (24). Briefly, deparaffinized sections were treated with Proteinase K for $40 \mathrm{~min}$. The hybridization was performed at $45^{\circ} \mathrm{C}$ for $75 \mathrm{~min}$ in a hybridization buffer containing Dual-DIG-labelled LNA probes miR-184 detection probe (probe sequence: ACCCTTATCAGTTCTCCGTCCA) or scramble-miR (probe sequence: GTGTAACACGTCTAT ACGCCCA) (Exiqon). Sections were blocked in a blocking buffer for 30 min and washed in PBS three times.

miR-184 target gene prediction algorithms. The presence of miR-184 target gene binding sites was analyzed using miRWalk, which collates data from multiple prediction programs as previously described (25).

Statistical analysis. Statistical analyses were performed using the SPSS software package. Results are expressed 

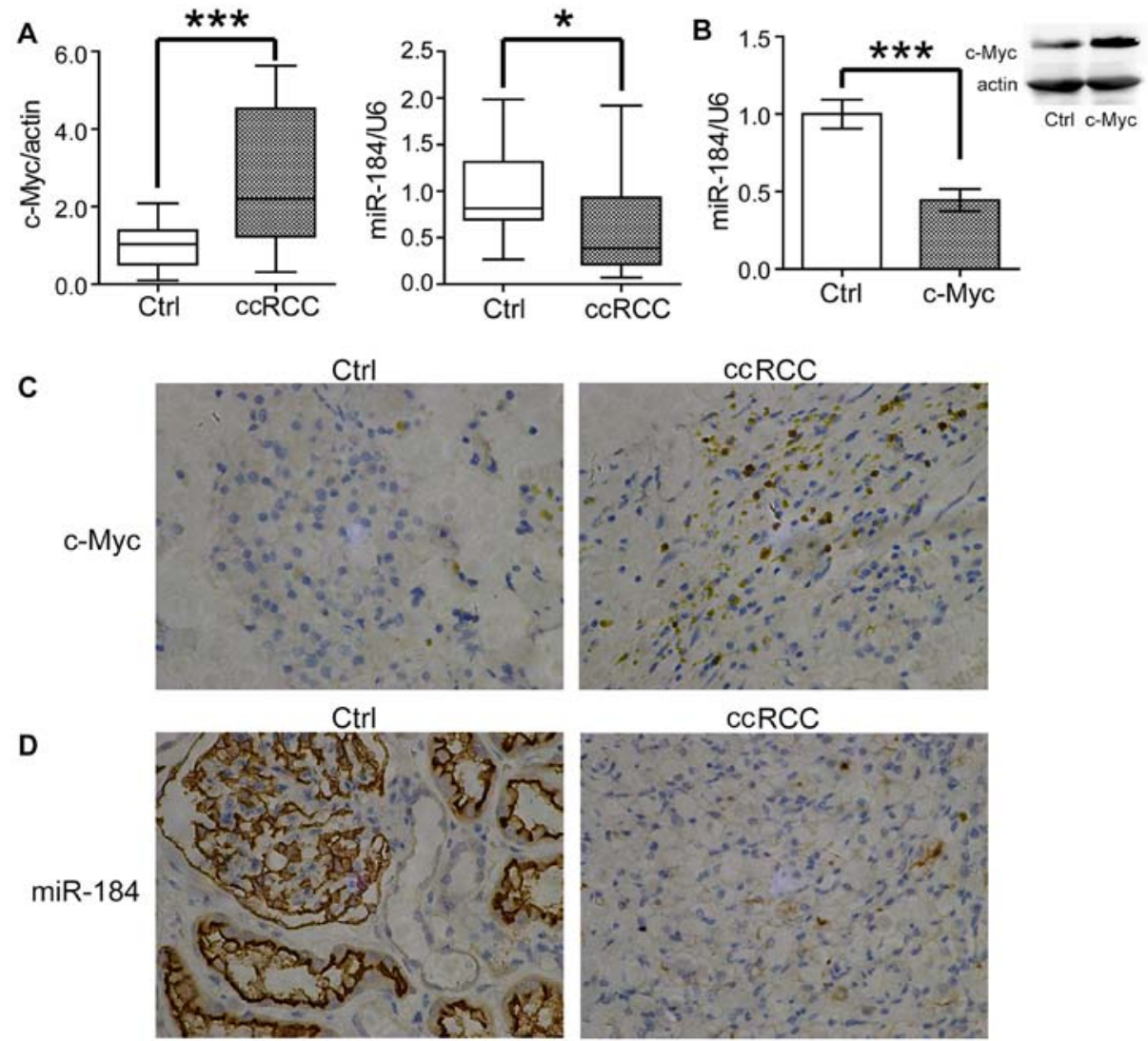

Figure 1. c-Myc negatively regulates miR-184 expression. (A) c-Myc and miR-184 expression in human ccRCC tissues compared to adjacent normal kidney tissue examined by real-time PCR. (B) miR-184 expression in cells transfected with control or c-Myc plasmids examined by real-time PCR. (Upper) Western blot analysis of c-Myc protein expression, examined $48 \mathrm{~h}$ after indicated plasmid transfection in 786-O cells. (C) c-Myc expression in ccRCC tissues examined by immunohistochemistry. (D) miR-184 expression in ccRCC tissues examined by in situ hybridization. Statistical significance was assessed by two-tailed Student's t-test. ${ }^{*} \mathrm{P}<0.05,{ }^{* * * *} \mathrm{P}<0.001$.

as mean \pm SD of at least three independent experiments. Statistical significance was assessed by two-tailed Student's t-test. Differences were considered statistically significant at $\mathrm{P}<0.05 . \mathrm{P}<0.05, \mathrm{P}<0.01$ and $\mathrm{P}<0.001$.

\section{Results}

c-Myc negatively regulates miR-184 expression in ccRCC. In order to investigate the relationship between c-Myc and miR-184, we first examined their expression in 50 matched pairs of human ccRCC samples and tumor adjacent normal kidney tissue. As shown in Fig. 1A, consistent with previous reports, the expression of c-Myc was greatly increased in ccRCC samples, whereas miR-184 was decreased comparing with the normal tissues. To confirm these results, we detected c-Myc and miR-184 expression by immunohistochemistry and in situ hybridization, respectively. Consistently, increased c-Myc and decreased miR-34a were observed in ccRCC samples (Fig. 1C and D). As c-Myc was reported to be a target of miR-184 $(12,14,15,19,20)$, we assume that the opposite expression profile between c-Myc and miR-184 may be a result of miR-184 targeting. However, we also noted that inhibition of MYCN, another member of MYC gene family, causes significantly upregulation of miR-184 (26). It is known that c-Myc functions as a transcription factor to influence the expression of a broad range of human genes involved in the progression of cell carcinogenesis (18). We wondered whether the downregulated expression of miR-184 could also be regulated by c-Myc. To test this possibility, we transfected c-Myc into the ccRCC cell line 786-O. Surprisingly, overexpression of c-Myc significantly downregulated the expression of miR-184 (Fig. 1B), which indicated miR-184 is a probable target of c-Myc in ccRCC cells.

miR-184 inhibits glucose metabolism in ccRCC cells. As ccRCC is featured by abnormal cell proliferation and metabolism, including increased glucose consumption, lactate production (6), we next wondered whether the abnormal proliferative and metabolic features in ccRCC cells could be improved by miR-184 overexpression. We transfected either the pre-miR negative control or the pre-miR-184 into the ccRCC cell lines 786-O and RCC4. As shown in Fig. 2A, transfection of pre-miR-184 led to a $\sim 3$-fold increase of the miR-184 expression level both in 786-O and RCC4 cells. Next, we measured glucose consumption and lactate production in these cells. As shown Fig. 2B, we observed significant glucose uptake reduction in pre-miR-184 transfected 786-O and RCC4 cells; in addition, lactate production were also decreased $\sim 30 \%$ in 786-O cells and $20 \%$ in RCC4 cells with miR-184 overexpression (Fig. 2C), indicating the metabolic features were improved by miR-184 overexpression. The effect of miR-184 on RCC cell proliferation was then analyzed. As shown in the MTT assay of Fig. 2D, the cell proliferation was markedly reduced by miR-184 overexpression in 786-O and RCC4 
A

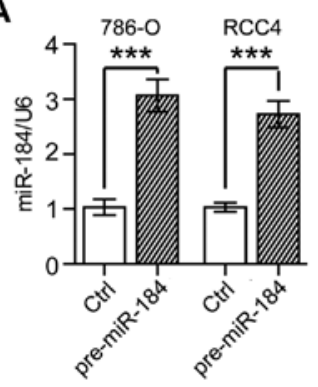

D
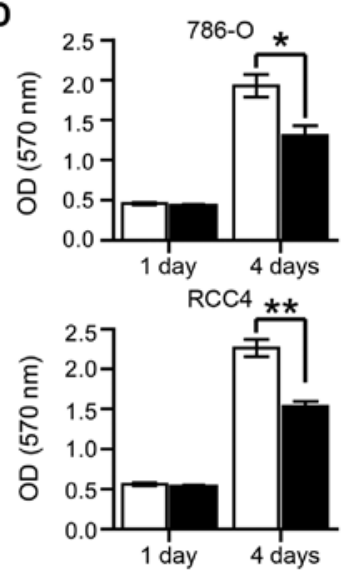

B

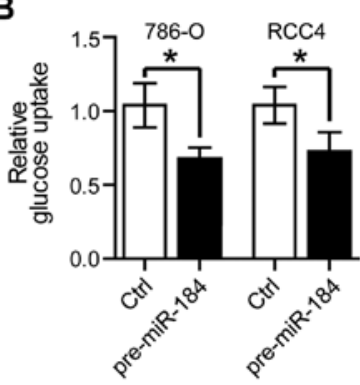

E
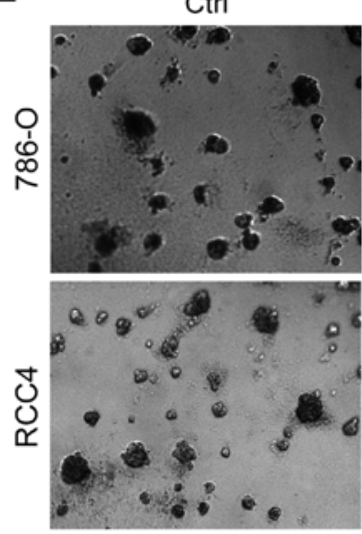

C

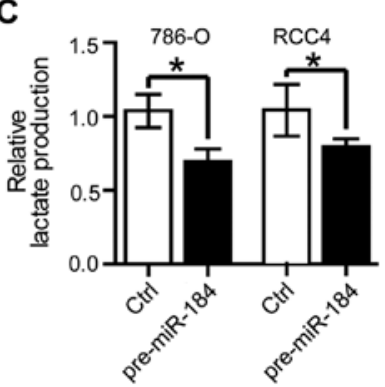

pre-miR-184

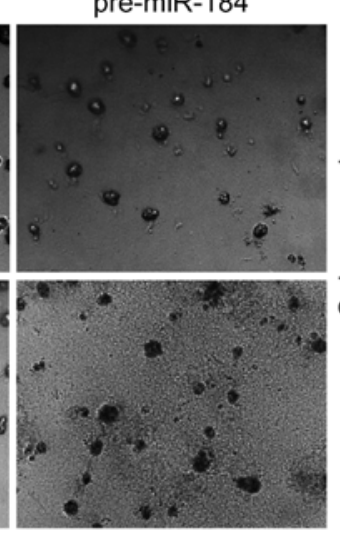

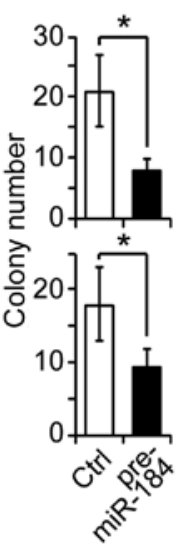

Figure 2. Overexpression of miR-184 alters glucose metabolism and proliferation in ccRCC cell line 786-O. (A) Overexpression of miR-184 in 786-O and RCC4 cells examined by real-time PCR. (B and C) control or pre-miR-184 were transfected in 786-O and RCC4 cells, respectively, $48 \mathrm{~h}$ after transfection, cells were harvest and subjected to glucose uptake (B) and lactate production (C) analysis. (D) miRNAs as indicated were transfected in 786-O and RCC4 cells, respectively, 24 and $96 \mathrm{~h}$ after transfection, cells were harvest and subjected to cell proliferation analysis by MTT, respectively. (E) Cell proliferation analysis by colony formation in 786-O and RCC4 cells. The statistical results are shown on the right. Statistical significance was assessed by two-tailed Student's t-test. ${ }^{*} \mathrm{P}<0.05,{ }^{* * *} \mathrm{P}<0.01,{ }^{* * *} \mathrm{P}<0.001$.

\section{A \\ hsa-miR-184 uGGGAAUAGUCAAGAGGCAGGU \\ $::|\quad|:|\quad|||||:|||| \mid$ \\ PKM2 3'-UTR WT tTTCCTGTGTGTACTCTGTCCA \\ PKM2 3'-UTR mutant tTTCCTGTGTGTACTCTGTGGU}

B
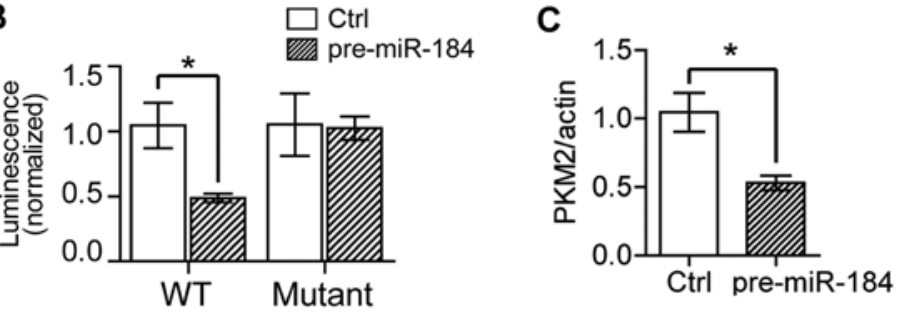

E
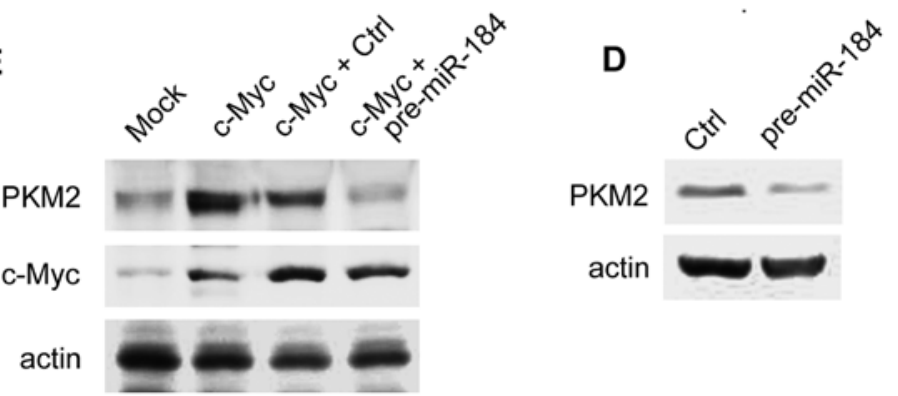

Figure 3. miR-184 specifically represses PKM2 expression. (A) Schema of binding site of miR-184 and mutation site in PKM2 3'UTR. (B) miRNAs were co-transfected with PKM2 WT or mutant 3'-UTR reporter and Renilla together with control or pre-miR-184 in 786-O cells, $48 \mathrm{~h}$ after transfection, cells were harvested and subjected to luciferase activity analysis. (C) PKM2 mRNA level examined by real-time PCR in 786-O cells $48 \mathrm{~h}$ after transfected with control or pre-miR-184. (D) Western blot analysis of endogenous PKM2 protein, examined $48 \mathrm{~h}$ after miRNAs transfection in 786-O cells. (E) Western blot analysis of c-Myc and PKM2 protein, examined $48 \mathrm{~h}$ after plasmid and/or miRNAs transfection in 786-O cells. Statistical significance was assessed by two-tailed Student's t-test. ${ }^{*} \mathrm{P}<0.05$. 
A

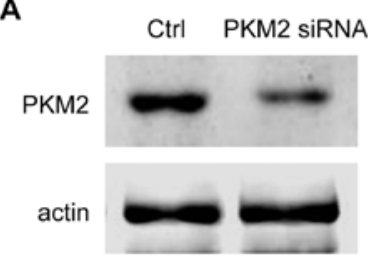

C

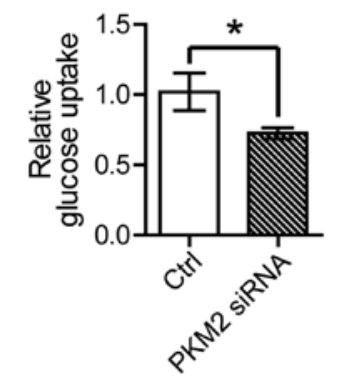

E

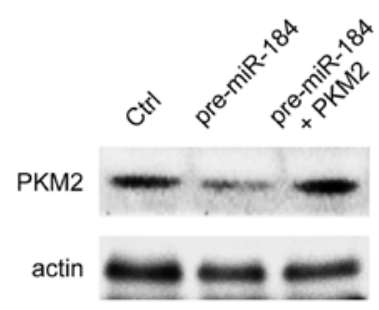

B

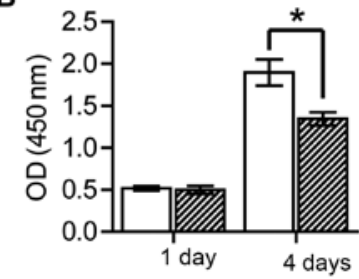

D

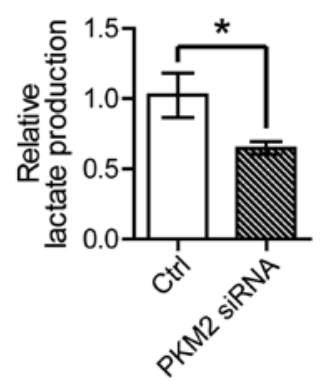

$\mathbf{F}$

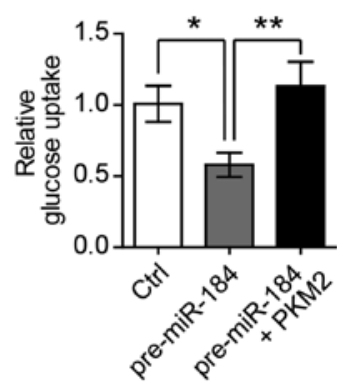

Figure 4. Knockdown of PKM2 suppresses the glycolysis and cell proliferation. (A) Western blot analysis of endogenous PKM2 protein, examined $48 \mathrm{~h}$ after indicated siRNA transfection in 786-O cells. (B) Cell proliferation analysis by MTT assay after transfection of control or PKM2 siRNAs. (C and D) Control or PKM2 siRNAs were transfected in 786-O cells, $48 \mathrm{~h}$ after transfection, cells were harvested and subjected to glucose uptake (C) and lactate production (D) analysis. (E) Western blot analysis of PKM2 protein, examined $48 \mathrm{~h}$ after indicated miRNAs and/or plasmid transfection in 786-O cells. (F) The cells in (E) were subjected to glucose uptake analysis. Statistical significance was assessed by two-tailed Student's t-test. ${ }^{*} \mathrm{P}<0.05,{ }^{* *} \mathrm{P}<0.01$.

cells on the fourth day after transfection. Similarly, miR-184 overexpression impaired colony formation of both 786-O and RCC4 cells (Fig. 2E). Thus, miR-184 inhibited ccRCC cell proliferation, glucose uptake and lactate production.

miR-184 specifically represses PKM2 expression. To uncover the mechanism of miR-184 contribution to ccRCC progression, we investigated putative miR-184 targets that directly regulate ccRCC metabolism and proliferation using bioinformatics methods. The bioinformatics analysis revealed that PKM2 (M2 isoform of pyruvate kinase) contains a potential miRNA response element (MRE) for miR-184 in its 3'-untranslated region (3'-UTR) (Fig. 3A). PKM2 is a 'glycolytic valve' in deciding the direction of glucose flux in glycolysis (27). It catalyzes the conversion of phosphoenolpyruvate (PEP) to pyruvate with concomitant production of ATP (28), which is independent of oxygen consumption. Thus, the high expression of PKM2 in tumor tissues helps tumor cells to grow rapidly even in hypoxic conditions (29). To determine whether PKM2 is indeed a target that is directly affected by miR-184, we examined the luciferase activity driven by PKM2-3'UTR containing the MRE sequence. As shown in Fig. 3B, in cells
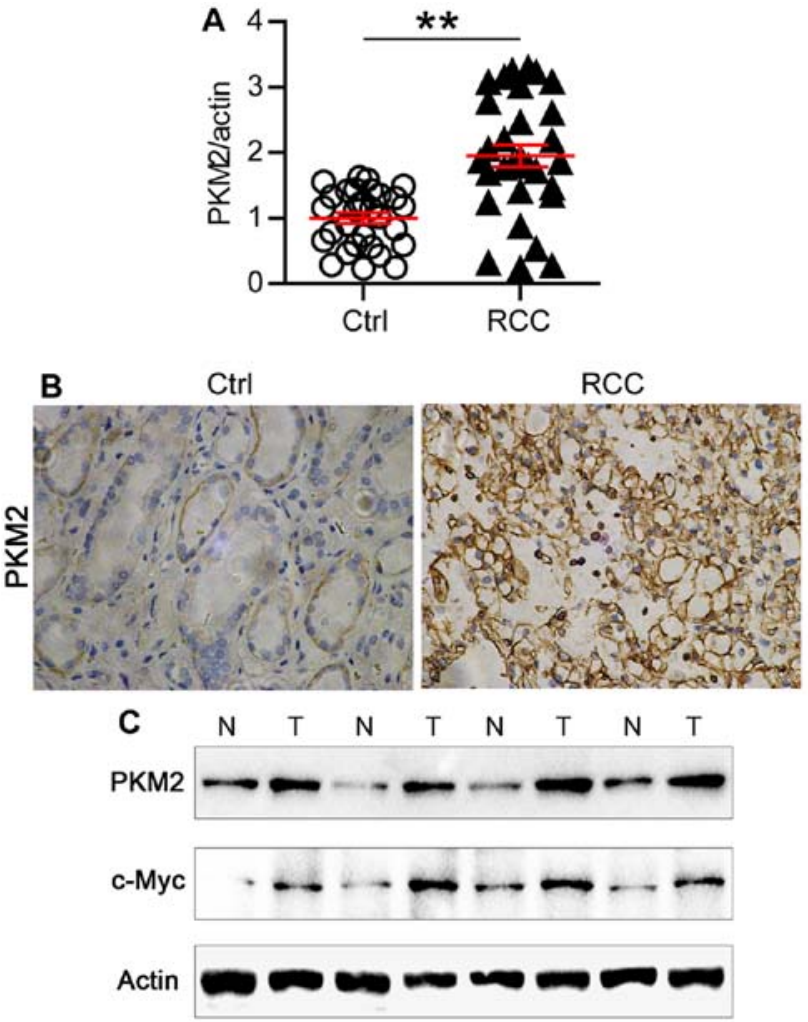

Figure 5. Expression of PKM2 is increased in renal clear cell carcinoma. (A) PKM2 expression in normal and ccRCC tissues determined by realtime PCR. (B) PKM2 expression in normal and ccRCC tissues examined by immunohistochemistry. (C) PKM2 and c-Myc expression in normal and ccRCC tissues determined by immunoblotting. N, normal sample; T, ccRCC tumor sample. Four pairs of samples are presented. Statistical significance was assessed by two-tailed Student's t-test. ${ }^{* * *} \mathrm{P}<0.001$.

transfected with promoter containing PKM2 WT MRE sequences, pre-miR-184 could significantly reduce the luminescence comparing with the control group. However, when the PKM2 MRE was mutated, miR-184 could not change the luciferase activity (Fig. 3A and B). To directly monitor the target effect of miR-184 on PKM2, we tested PKM2 expression both at mRNA level and protein expression level. In accordance, pre-miR-184 decreased PKM2 expression by $50 \%$ at mRNA level (Fig. 3C), and repressed PKM2 protein expression obviously (Fig. 3D). We also examined the effects of c-Myc overexpression on PKM2 expression. As shown in Fig. 3E, PKM2 expression was enhanced by c-Myc overexpression. However, when c-Myc and pre-miR-184 were co-expressed, the upregulated PKM2 by c-Myc overexpression was abolished in response to miR-184 overexpression, suggesting that c-Myc indeed increased PKM2 expression by inhibiting miR-184 expression. In brief, our data indicated PKM2 is a new target of miR-184 in ccRCC cells.

Knockdown of PKM2 suppressed the glycolysis and cell proliferation. After discovering PKM2 as a direct target of miR-184, we next asked whether the altered metabolic and proliferative features in ccRCC were the results of increased PKM2 level, as the miR-184 was downregulated. To test this hypothesis, we transfected PKM2 siRNA into 786-O cells to lower PKM2 expression level (Fig. 4A). Firstly we analyzed 


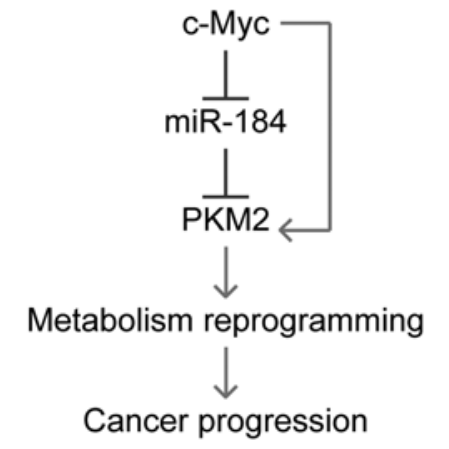

Figure 6. Schematic model of c-Myc-induced PKM2 upregulation by repression of miR-184. A mechanism diagram of c-Myc modulates glucose metabolism via regulation of miR-184/PKM2 pathway in clear-cell renal cell carcinoma.

the proliferation of PKM2 knockdown cells, and found a profound decrease in proliferative cell numbers by MTT on the fourth day post-PKM2 siRNA transfection (Fig. 4B). Furthermore, consistent with our expectations, knockdown of PKM2 remarkably decreased glucose uptake and lactate production in 786-O cells (Fig. 4C and D). Furthermore, we found pre-miR-184 overexpression in 786-O cells resulted in impaired glucose uptake, which could be rescued by PKM2 overexpression. This result, therefore, suggested that miR-184 inhibited glycolysis in a PKM2-dependent manner (Fig. 4E and F). These data supported the conclusion that knockdown of PKM2 inhibits abnormal cell proliferation and metabolism in ccRCC cell line.

Expression of PKM2 is increased in ccRCC. Finally, we examined the expression of PKM2 in human ccRCC samples. As shown in Fig. 5A, the expression of PKM2 mRNA doubled in the ccRCC samples compared with the normal tissue. Consistently, high expression of PKM2 protein level was also observed in ccRCC samples examined by immunohistochemistry (Fig. 5B). Then we analyzed the protein expression level of PKM2 and c-Myc in paired ccRCC samples. Strikingly, increased expression of both PKM2 and c-Myc were observed in one same ccRCC sample comparing with paired normal tissue (Fig. 5C). Thus, our results indicated that the high expression of PKM2 in ccRCC tissues might be a reason leading to hyperproliferation and metabolic disorder of ccRCC.

\section{Discussion}

ccRCC is one of the most malignant tumors worldwide. It consists of several histologic subtypes, including clear cell, papillary, chromophobe and collecting duct (3). Each subtype is different in molecular profiling, and ccRCC is the most common and aggressive one. One of the most important difficulties for ccRCC treatment is its resistance to traditional therapies $(4,30)$. Therefore, the mechanism study in ccRCC progression is critical for uncovering the putative molecular targets for ccRCC therapy.

To date, many proteins and RNAs have been reported to take part in the progression of ccRCC, such as c-Myc, VHL, miR-34 and miR-184 (7,10,21,31). However, the exact mecha- nism was not clarified. In the present study, we identified that miR-184 could be regulated by c-Myc, which is contrary to general opinion that c-Myc is a target of miR-184.

miR-184 has been reported to be downregulated in many cancer types, including ccRCC. In this study, we found overexpression of pre-miR-184 could reduce ccRCC cell glucose consumption, lactate production and cell proliferation. Further analysis by computer bioinformatics revealed that PKM2 is a target of miR-184. Both the mRNA and protein level of PKM2 was markedly reduced in response to miR-184 overexpression. Most importantly, the PKM2 expression level was indeed increased in ccRCC samples, which is totally reverse to the decreased miR-184 expression level. This result indirectly verified that PKM2 is a target of miR-184 in human ccRCC samples.

PKM2 is a key regulator of the metabolic fate of the glycolytic intermediates, its high expression in many cancers favored the cancer cells to proliferate rapidly even in anaerobic conditions. We found that knockdown of PKM2 in ccRCC cells, inhibited the rapid proliferation, high glucose consumption and high lactate production to a certain extent, which indicated metabolic reprogramming in ccRCC cells. In summary, in the present study, we identified PKM2 as a new target of miR-184, and that its expression can be inhibited by c-Myc. As a result, PKM2 expression can be upregulated by c-Myc overexpression. Knockdown the high PKM2 expression profile in ccRCC leads to metabolism reprogramming in these cancer cells, and then inhibits cancer progression of ccRCC (Fig. 6). Our findings shed new light on ccRCC molecular study and provide a new and solid basis for developing ccRCC therapy.

\section{Acknowledgements}

The design and conduct of the project was supported by the Award Number 81402084, 81272841, 81472378 from the National Natural Science Foundation of China and the Award Number PYXJS16-008 from the Incubating Program for Clinical Research and Innovation of Renji Hospital.

\section{References}

1. Chow WH, Dong LM and Devesa SS: Epidemiology and risk factors for kidney cancer. Nat Rev Urol 7: 245-257, 2010.

2. Majer W, Kluzek K, Bluyssen H and Wesoły J: Potential approaches and recent advances in biomarker discovery in clearcell renal cell carcinoma. J Cancer 6: 1105-1113, 2015.

3. Cohen HT and McGovern FJ: Renal-cell carcinoma. N Engl J Med 353: 2477-2490, 2005.

4. Amato RJ: Chemotherapy for renal cell carcinoma. Semin Oncol 27: 177-186, 2000.

5. Beck SD, Patel MI, Snyder ME, Kattan MW, Motzer RJ, Reuter VE and Russo P: Effect of papillary and chromophobe cell type on disease-free survival after nephrectomy for renal cell carcinoma. Ann Surg Oncol 11: 71-77, 2004.

6. Massari F, Ciccarese C, Santoni M, Brunelli M, Piva F, Modena A, Bimbatti D, Fantinel E, Santini D, Cheng L, et al: Metabolic alterations in renal cell carcinoma. Cancer Treat Rev 41: 767-776, 2015.

7. Linehan WM, Srinivasan R and Schmidt LS: The genetic basis of kidney cancer: A metabolic disease. Nat Rev Urol 7: 277-285, 2010.

8. Gossage L, Eisen T and Maher ER: VHL, the story of a tumour suppressor gene. Nat Rev Cancer 15: 55-64, 2015. 
9. Cancer Genome Atlas Research Network: Comprehensive molecular characterization of clear cell renal cell carcinoma Nature 499: 43-49, 2013.

10. Tang SW, Chang WH, Su YC, Chen YC, Lai YH, Wu PT, Hsu CI, Lin WC, Lai MK and Lin JY: MYC pathway is activated in clear cell renal cell carcinoma and essential for proliferation of clear cell renal cell carcinoma cells. Cancer Lett 273: 35-43, 2009.

11. Ma L and Qu L: The function of microRNAs in renal development and pathophysiology. J Genet Genomics 40: 143-152, 2013

12. Wong TS, Liu XB, Wong BY, Ng RW, Yuen AP and Wei WI Mature miR-184 as potential oncogenic microRNA of squamous cell carcinoma of tongue. Clin Cancer Res 14: 2588-2592, 2008.

13. Foley NH, Bray IM, Tivnan A, Bryan K, Murphy DM, Buckley PG, Ryan J, O'Meara A, O'Sullivan M and Stallings RL: MicroRNA-184 inhibits neuroblastoma cell survival through targeting the serine/threonine kinase AKT2. Mol Cancer 9: 83, 2010.

14. Zhen Y, Liu Z, Yang H, Yu X, Wu Q, Hua S, Long X, Jiang Q, Song Y, Cheng C, et al: Tumor suppressor PDCD4 modulates miR-184-mediated direct suppression of C-MYC and BCL2 blocking cell growth and survival in nasopharyngeal carcinoma. Cell Death Dis 4: e872, 2013

15. Wu GG, Li WH, He WG, Jiang N, Zhang GX, Chen W, Yang HF, Liu QL, Huang YN, Zhang L, et al: Mir-184 post-transcriptionally regulates SOX7 expression and promotes cell proliferation in human hepatocellular carcinoma. PLoS One 9: e88796, 2014.

16. Leng HM, Qian WP, Zhou L, Zhai QN, Li XX, Guan ZC, Gui YT and Cai ZM: Abnormal expression and significance of MIR-184 in human renal carcinoma. Beijing Da Xue Xue Bao 43: 509-513, 2011 (In Chinese).

17. Su Z, Chen D, Li Y, Zhang E, Yu Z, Chen T, Jiang Z, Ni L, Yang S, Gui Y, et al: microRNA-184 functions as tumor suppressor in renal cell carcinoma. Exp Ther Med 9: 961-966, 2015.

18. Lebofsky R and Walter JC: New Myc-anisms for DNA replication and tumorigenesis? Cancer Cell 12: 102-103, 2007.

19. Lin TC, Lin PL, Cheng YW, Wu TC, Chou MC, Chen CY and Lee H: MicroRNA-184 deregulated by the microRNA-21 promotes tumor malignancy and poor outcomes in non-small cell lung cancer via targeting CDC25A and c-Myc. Ann Surg Oncol 22 (Suppl 3): 1532-1539, 2015.
20. Liu Z, Mai C, Yang H, Zhen Y, Yu X, Hua S, Wu Q, Jiang Q, Zhang Y, Song X, et al: Candidate tumour suppressor CCDC19 regulates miR-184 direct targeting of C-Myc thereby suppressing cell growth in non-small cell lung cancers. J Cell Mol Med 18: 1667-1679, 2014

21. Shroff EH, Eberlin LS, Dang VM, Gouw AM, Gabay M, Adam SJ, Bellovin DI, Tran PT, Philbrick WM, Garcia-Ocana A, et al: MYC oncogene overexpression drives renal cell carcinoma in a mouse model through glutamine metabolism. Proc Natl Acad Sci USA 112: 6539-6544, 2015.

22. Liu W, Cao H, Ye C, Chang C, Lu M, Jing Y, Zhang D, Yao X, Duan Z, Xia H, et al: Hepatic miR-378 targets p110 $\alpha$ and controls glucose and lipid homeostasis by modulating hepatic insulin signalling. Nat Commun 5: 5684, 2014.

23. Jo HS, Kang KH, Joe CO and Kim JW: Pten coordinates retinal neurogenesis by regulating Notch signalling. EMBO J 31: 817-828, 2012.

24. Jia LF, Wei SB, Gong K, Gan YH and Yu GY: Prognostic implications of micoRNA miR-195 expression in human tongue squamous cell carcinoma. PLoS One 8: e56634, 2013.

25. Dweep H, Sticht C, Pandey P and Gretz N: miRWalk - database: Prediction of possible miRNA binding sites by 'walking' the genes of three genomes. J Biomed Inform 44: 839-847, 2011.

26. Chen Y and Stallings RL: Differential patterns of microRNA expression in neuroblastoma are correlated with prognosis, differentiation, and apoptosis. Cancer Res 67: 976-983, 2007.

27. Iqbal MA, Gupta V, Gopinath P, Mazurek S and Bamezai RN: Pyruvate kinase M2 and cancer: An updated assessment. FEBS Lett 588: 2685-2692, 2014.

28. Gupta V and Bamezai RN: Human pyruvate kinase M2: a multifunctional protein. Protein Sci 19: 2031-2044, 2010.

29. Wong N, Ojo D, Yan J and Tang D: PKM 2 contributes to cancer metabolism. Cancer Lett 356: 184-191, 2015.

30. Lane BR, Rini BI, Novick AC and Campbell SC: Targeted molecular therapy for renal cell carcinoma. Urology 69: 3-10, 2007.

31. Redova M, Svoboda M and Slaby O: MicroRNAs and their target gene networks in renal cell carcinoma. Biochem Biophys Res Commun 405: 153-156, 2011. 